\title{
TANINOS Y FENOLES EN LA FERMENTACIÓN IN VITRO DE LEÑOSAS FORRAJERAS TROPICALES ${ }^{1}$
}

\author{
René Pinto-Ruiz ${ }^{2}$, David Hernández-Sánchez ${ }^{3}$,Luis Ramírez-Avilés ${ }^{4}$,Carlos Alfredo Sandoval-Castro ${ }^{4}$, Mario \\ Cobos-Peralta ${ }^{3}$,Heriberto Gómez-Castro ${ }^{2}$
}

\begin{abstract}
RESUMEN
Taninos y fenoles sobre la fermentación in vitro de leñosas forrajeras tropicales. Con el objetivo de determinar la actividad biológica de factores antinutricionales en nueve follajes y cinco frutos arbóreos forrajeros de la región central del estado de Chiapas, México, se llevó a cabo el presente trabajo durante el año 2006. Los factores antinutricionales estudiados fueron polifenoles totales y taninos condensados. El método utilizado fue a través de la técnica de producción de gas in vitro en medio rico en nitrógeno y la adición o no de Polietilenglycol (PEG-4000). Se utilizó un diseño experimental completamente al azar, así como regresiones y correlaciones lineales simples. La composición química mostró una amplia variación entre especies. El uso de PEG aumentó significativamente $(\mathrm{p}<0,05)$ la producción de gas en los follajes de L. collinsii, G. ulmifolia, A. pennatula y A. milleriana y en los frutos A. milleriana, G. ulmifolia y $L$. collinsii. En el follaje, los incrementos en la producción de gas estuvieron asociados inversamente a los altos contenidos de fenoles y taninos condensados de las especies ( $\mathrm{r}-0,68$, $\mathrm{p}<0,04$ y r $-0,67, \mathrm{p}<0,03$ respectivamente). En los frutos, no se encontró relación entre fenoles y taninos y la producción de gas, pero si se encontró asociación inversa a las fracciones de fibra $(F D N=$ r -0,97, p<0,06 y FDA $=$ r -0,94, p<0,01). Resaltan las altas concentraciones de factores antinutricionales presentes en follajes de algunas especies y la importancia del PEG para demostrar la alta actividad de éstos.
\end{abstract}

Palabras clave: Taninos condensados, polifenoles totales, producción de gas, árboles forrajeros, México.

\begin{abstract}
Tannins and phenols on in vitro fermentation of tropical fodder trees. The objective of this study was to determine the biological activity of anti-nutritional factors present in nine foliage and five fruit samples from forage-trees of Central Chiapas, México, in 2006. The anti-nutritional factors studied were total polyphenols and condensed tannins. The experimental design was completely-randomized, and statistics was done by regression and correlation analyzes. The method used was in vitro gas production in an $\mathrm{N}$-enriched solution, and the addition of Poly-ethylene glycol (PEG-4000). Chemical composition and increase in gas production of samples (over 24 hours and total) were analyzed. Chemical composition varied wirdely among species. PEG raised significantly gas production $(\mathrm{p}<0.05)$ in foliage of $L$. collinsii, G. ulmifolia, A. pennatula and A. milleriana and in fruits of A. milleriana, G. ulmifolia y L. collinsii. In foliage samples, the increase in gas production was negatively related to high phenols and condensed tannin content of the species (FDN= r= $-0.68, \mathrm{p}<0.04$ and $\mathrm{r}-0.67, \mathrm{p}<0.03$ respectively). In fruits, there was not relation between phenol and tannin content and gas production, but there was an inverse relation with fiber contents $(\mathrm{FDN}=\mathrm{r}-0.97, \mathrm{p}<0.06$ and $\mathrm{FDA}=\mathrm{r}-0.94$, $\mathrm{p}<0.01)$. The forage-trees studied contain high levels of anti-nutritional factors, particularly in their foliage and PEG is useful to study their anti-nutritional activity.
\end{abstract}

Key words: Condensed tannins, total poly-phenols, gas production, forage trees, Mexico.

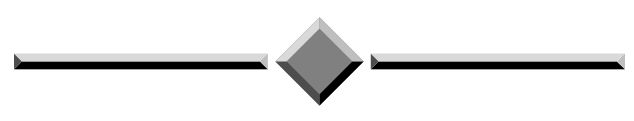

1 Recibido: 16 de agosto, 2007. Aceptado: 20 de marzo, 2009. Parte del proyecto "Identificación de árboles forrajeros del trópico seco de Chiapas", México.

2 Facultad de Ciencias Agronómicas. Universidad Autónoma de Chiapas. Carretera Villaflores-Ocozocoautla km 7.5. AP 30470, México. Tel (+965) 6521477.pinto_ruiz@yahoo.com.mx; hgomezc@yahoo.com

3 Colegio de Postgraduados. Programa de Ganadería. Carretera México-Texcoco Km. 36.5. C. P. 56230 Montecillo, Texcoco, Estado de México, México. Tel. (+595) 95 20200, ext. 1727. sanchezd@colpos.mx

4 Facultad de Medicina Veterinaria y Zootecnia. Universidad Autónoma de Yucatán. Apdo. 4-116 Itzimna, Mérida, Yucatán, 97100, México. raviles@tunku.uady.m; csandoval@tunku.uady.mx 


\section{INTRODUCCIÓN}

Muchas de las especies arbóreas estudiadas como recurso forrajero en los trópicos poseen un follaje generalmente rico en proteína y minerales y se utilizan como suplemento durante la época de verano en animales alimentados a base de pastos de pobre calidad y/o residuos de cosecha (Devendra 1990). Sin embargo, existen trabajos que evidencian que la presencia de compuestos antinutricionales en estas plantas, inhiben la acción de enzimas y la actividad de los microorganismos en el rumen, limitan la degradación de nutrientes y reducen, la producción de ácidos grasos como productos finales de la degradación (Ramana et al. 2000, Salem et al. 2006). Estos compuestos presentes en las especies arbóreas pueden ejercer efectos detrimentales sobre la digestibilidad, el consumo y por tanto sobre el comportamiento animal (Tolera et al. 1997). Por ello, la determinación de la presencia y actividad biológica de estos compuestos fenólicos ha sido estudiada en años recientes, así como las alternativas para mejorar su uso dentro de la alimentación animal. Sin embargo, aún hace falta cuantificar el contenido químico y su efecto sobre las características de degradación de un gran número de especies arbóreas utilizadas en diversas regiones del mundo, donde su uso como parte de la dieta animal es importante. Por tanto, es esencial que se utilicen o desarrollen técnicas que contribuyan a medir la actividad de los compuestos antinutricionales que estas especies contienen.

El uso de la técnica de producción de gas in vitro en conjunto con agentes inhibidores ha sido establecido para el estudio de factores antinutricionales en especies arbóreas (Theodorou et al. 1994). Se ha propuesto el uso del Polietilenglycol (PEG-4000) para detectar la actividad biológica de compuestos fenólicos en las plantas (Makkar et al. 1995). El PEG es un agente que neutraliza el efecto de los taninos sobre la fermentación ruminal; por lo que, un incremento en la producción de gas in vitro y en los parámetros de degradación cuando se suministra PEG indicaría la actividad de taninos en los alimentos (Getachew et al. 1998).

El objetivo de este trabajo fue conocer el efecto del contenido de taninos y fenoles totales en follajes y frutos de algunas especies arbóreas sobre la producción de gas in vitro con la utilización de PEG como herramienta para detectar actividad antinutricional en los alimentos de uso animal.

\section{MATERIALES Y MÉTODOS}

Características y manejo de las muestras arbóreas. El experimento fue conducido en el Laboratorio de Nutrición Animal de la Facultad de Medicina Veterinaria y Zootecnia de la Universidad Autónoma de Yucatán, México durante el año 2004. Con base en el follaje de Guazuma ulmifolia, Gliricidia sepium, Leucaena collinsii, Diphysa robinoides, Erythrina goldmanii, Genipa americana, Phitecellobium dulce, Acacia pennatula y Acacia milleriana; y el fruto de Acacia milleriana, Enterolobium cyclocarpum, Leucaena collinsii, Guazuma ulmifolia y Ficus glabrata. Estas especies forrajeras fueron seleccionadas en un trabajo previo (Pinto et al. 2002) como las de mayor importancia del trópico seco del estado de Chiapas, México. Muestras de cada una de éstas fueron cosechadas manualmente durante la primavera (época de secas) de distintos árboles. El muestreo se realizó a través de simulación de pastoreo, lo cual consistió en tomar aquellas partes de la planta que el animal selecciona durante el pastoreo (Fick et al. 1979). Las muestras fueron almacenadas bajo refrigeración dos días a $18{ }^{\circ} \mathrm{C}$ y secadas en una estufa de aire forzado Modelo $632 / 2$, a $60{ }^{\circ} \mathrm{C}$ durante dos horas. Una vez secas, las muestras fueron procesadas en un molino tipo Wiley con una criba de $1 \mathrm{~mm}$, utilizándose hielo seco durante el proceso. Las muestras fueron incubadas con y sin PEG. Las muestras se incubaron con $1 \mathrm{~g}$ por cada 0,5 $\mathrm{g}$ de follaje o fruto de cada especie.

Tratamientos, diseño experimental y análisis estadístico. Se evaluaron nueve tratamientos (especies) correspondientes a follajes y cinco tratamientos correspondientes a frutos, cada uno de ellos fue repetido cuatro veces. Las diferencias entre las muestras respecto a su composición química, producción de gas a $24 \mathrm{~h}$ y de las variables de degradación fueron analizadas con base en un análisis de varianza para un diseño experimental completamente al azar. Las diferencias de medias fueron comparadas utilizando la prueba de Tukey (Steel y Torrie 1988). La relación entre la composición química de las leñosas y la producción de gas in vitro a 24 h fue obtenida a través de correlaciones y regresiones simples. Todos los análisis se realizaron con base en el procedimiento GLM del paquete estadístico SAS (SAS 1999).

Análisis químico de las muestras. Tanto el follaje como el fruto de las especies bajo estudio se analizó para conocer los contenidos de Proteína Cruda (PC), 
Cenizas (Ce) y Materia Orgánica (MO) siguiendo la metodología descrita por la A.O.A.C. (1990), las Fracciones de Fibra (Fibra Detergente Neutro (FDN) y Fibra Detergente Ácida (FDA), según Van Soest y Robertson (1991), y presencia de Fenoles Totales (FT) y Taninos Condensados (TC) según Makkar (2003).

Producción de gas in vitro. La técnica empleada fue la descrita por Theodorou et al. (1994) con base en un transductor (Druck Incorporated DPI 701555 in $\mathrm{H}_{2} 0 / 20 \mathrm{psi}$ g), para medir la presión generada por la producción de $\mathrm{CO}_{2}$ y jeringas de plástico para medir el volumen de gas (milímetros).

Preparación del medio. La preparación del medio según Menke y Steingass (1988) consideró diversos componentes: Solución de Microminerales, Solución Buffer, Solución de Macrominerales, Solución Reductora, Cisteina, Indicador Anaerobio y Resazurina.

Obtención del inóculo. Los animales donadores (dos bovinos cebú de un peso vivo de $320 \mathrm{~kg} \pm 18,5$ $\mathrm{kg}$ y una edad de tres años) poseían cánulas ruminales y por 12 horas estuvieran en ayuno y sin agua para obtener la muestra de ingesta. La dieta de los animales consistió en pasto fresco (Cynodon pletostachyus) y un concentrado con $87 \%$ de Materia Seca y $12 \%$ de Proteína Cruda, a base de sorgo, soya y la mezcla mineral comercial Microfos PR-9® (MNA de México). Las muestras de contenido ruminal se obtuvieron de la parte ventral del rumen y fueron colocadas en recipientes sellados para su protección del aire. Posteriormente, las muestras fueron coladas, con un colador de malla colocado dentro de un vaso de precipitado y manteniendo el flujo de $\mathrm{CO}_{2}$, después se procedió a licuar los sólidos remanentes en la malla en una licuadora, a la cual se le agregó la solución buffer durante 20 segundos a $1.500 \mathrm{rpm}$; se mantuvo el flujo de $\mathrm{CO}_{2}$ para evitar la entrada de aire por efecto del licuado. Se filtró de nuevo el líquido obtenido y se adicionó al líquido ruminal filtrado anteriormente. Esta mezcla fue el inóculo del líquido ruminal que finalmente se adicionó a cada botella. El inóculo de líquido ruminal se mantuvo bajo $\mathrm{CO}_{2}$ en una placa de calentamiento a $39^{\circ} \mathrm{C}$, hasta que fue utilizado.

Método de infusión. La metodología consideró los siguientes pasos:

1. Se utilizaron botellas de vidrio de $100 \mathrm{ml}$ de capacidad, en cada una se introdujo $0,5 \mathrm{~g}$ de cada una de las muestras de las arbóreas evaluadas (por cuadruplicado) con y sin PEG, tanto de follaje como de fruto, y un juego de blancos para cada uno de éstos. Se tomó una submuestra de cada especie para determinar Materia Seca (MS).

2. Se mezcló el medio preparado inicialmente con el inóculo, manteniendo un flujo continuo de $\mathrm{CO}_{2}$. De esta mezcla se adicionaron $60 \mathrm{ml}$ con una jeringa de plástico a cada botella.

3. Antes de sellar las botellas se continuó con el flujo de $\mathrm{CO}_{2}$ para asegurar la anaerobiosis.

4. Una vez selladas las botellas con la gárgola, se procedió a pinchar los tapones con una aguja. En este momento se colocaron todas las muestras en la incubadora y se empezó a contar el tiempo de incubación e iniciar las lecturas a los horarios correspondientes.

El tiempo cero para el cultivo fue cuando las botellas fueron selladas y pinchadas. Los tiempos de medición postinfusión fueron tres, seis, nueve, 12, 15, $18,21,24,30,36,48,60,72$ y 96 h. En cada botella se hicieron las mediciones a los diferentes tiempos.

El proceso de filtrado de la solución final de cada botella (después de 96 h) se hizo con papel filtro grueso comercial (correspondiente al papel filtro Whatman No. 2), previamente identificado y pesado. Una vez filtradas, las muestras fueron secadas a una temperatura de $60{ }^{\circ} \mathrm{C}$ por $12 \mathrm{~h}$, para determinar MS y por diferencia con el material inicialmente incubado calcular la MS degradada.

Mediciones por producción de gas in vitro. Se consideró la producción acumulada in vitro de gas a 24 h y las variables de producción de gas de las especies arbóreas forrajeras en estudio. Para efecto de comparar los incrementos (\%) en la producción de gas en las especies tratadas con PEG respecto a las no tratadas a las 24 horas de incubación se utilizó la fórmula propuesta por Khazaal et al. (1994): Incremento (\%)= ((volumen de gas con PEG) - (volumen de gas sin PEG)) / (volumen de gas sin PEG)*100.

Procesamiento de los datos. Los datos de los perfiles de producción de gas de 0 a 96 h se procesaron con base en el modelo sin el tiempo de retraso (Theodorou et al. 1994) para derivar las variables de degradación a, b y c:

$$
\mathrm{Y}=\mathrm{a}+\mathrm{b}(1-\text { exp-ct })
$$

Donde:

$\mathrm{Y}=$ Producción de gas en el tiempo t.

$\mathrm{a}, \mathrm{b}=$ Constantes de la ecuación.

$\mathrm{c}=$ Tasa de producción del gas. 


\section{RESULTADOS Y DISCUSIÓN}

Composición química de las arbóreas. Respecto a follajes (Cuadro 1), los valores más altos de PC $(\mathrm{p}<0,05)$ correspondieron a las especies $G$. sepium y E. goldmanii. A pennatula presentó los valores más altos $(\mathrm{p}<0,05)$ de FDN, FDA, FT y TC en comparación con las otras especies. Similarmente, G. ulmifolia y A. milleriana presentaron valores altos $(\mathrm{p}<0,05)$ de FT y TC. Con referencia a frutos (Cuadro 2), sobresalieron por su valor proteico $(\mathrm{p}<0,05)$ L. collinsii, E. cyclocarpum y $F$. glabrata. F. glabrata presentó los más altos valores de fracciones de fibra $(\mathrm{p}<0,05)$, en tanto, A. milleriana presentó los mayores valores de FT y $L$. collinsii de TC ( $<<0,05)$. En general, se encontró una amplia variación en la composición química entre las diferentes especies así como entre sus componentes (follajes y frutos).

La concentración promedio de PC en la mayoría de las especies evaluadas, tanto en follaje como fruto, superó el contenido de PC de los pastos más importantes de la región, el cual es reportado con valores promedios anuales de 6,5 \% (Pinto 2002), así también, se encuentran dentro del rango considerado por Norton (1994) como los normales para especies arbóreas (12$30 \%$ ). Considerando la concentración de PC, el $7 \%$ de los follajes y el $60 \%$ de los frutos pueden satisfacer los requerimientos de mantenimiento y producción de cabras y ovinos (Mtengeti y Mhelela 2006). Solo G. americana podría no proveer los niveles mínimos de amonio requerido para rumiantes (Norton 1994) lo que podría representar una limitante en su empleo.

Los valores de las fracciones fibrosas obtenidas se encuentran dentro de los rangos reportados en los trabajos de Ramana et al. (2000), Sosa et al. (2004), García y Medina (2006) y Tedonkeng et al. (2007) y podrían estar atribuidos a las características morfológicas del árbol, sitio de crecimiento de la planta, edad, suelo y a las diferencias de lignificación de las paredes celulares (Makkar y Singh 1991). Los valores de FT y TC fueron mayores en follajes que en frutos (13,7 y 34,1 vs 9,6 y $13,5 \mathrm{~g} / \mathrm{kg} / \mathrm{MS}$ respectivamente), pudiéndose atribuir dichas diferencias a las condiciones ambientales, estado fisiológico de la planta y a la nutrición de la misma (Makkar y Singh 1991). Las diferencias en la composición química de los follajes y frutos evaluados podrían dar como resultado diferencias en el aporte de nitrógeno degradable en rumen y energía para síntesis de proteína microbiana, así como en el flujo de nutrientes a sitios post-ruminales para la utilización animal (Mupangwa et al. 1997).

Producción de gas in vitro a $\mathbf{2 4} \mathbf{h}$. Los resultados presentados en este trabajo están basados en el contenido de MS de las muestras, y presentados como las medias de las repeticiones empleadas en las mediciones. La producción de gas (media de cuatro réplicas) con y sin la adición de PEG fue calculada como ml por 500 mg/MS. El tratamiento con PEG aumentó significativamente $(\mathrm{p}<0,05)$ el volumen de gas $(\mathrm{ml})$ a $24 \mathrm{~h}$ en las especies $L$. collinsii, G. ulmifolia, A. pennatula y A. milleriana para

Cuadro 1. Composición química ${ }^{1}$ ( $\mathrm{g} / \mathrm{kg}_{\mathrm{g}}$ de materia seca) del follaje de leñosas forrajeras del trópico seco del estado de Chiapas, México, 2004.

\begin{tabular}{llllllll}
\hline \multicolumn{1}{c}{ Especies } & \multicolumn{1}{c}{ PC } & \multicolumn{1}{c}{ Ce } & MO & FDN & FDA & FT & TC \\
\hline Guazuma ulmifolia & $104,10 \mathrm{c}$ & $137,91 \mathrm{a}$ & $862,00 \mathrm{~d}$ & $425,20 \mathrm{c}$ & $295,72 \mathrm{~b}$ & $28,01 \mathrm{~b}$ & $47,11 \mathrm{~b}$ \\
Gliricidia sepium & $238,11 \mathrm{a}$ & $106,42 \mathrm{bc}$ & $894,10 \mathrm{~b}$ & $385,19 \mathrm{~cd}$ & $247,65 \mathrm{~d}$ & $3,03 \mathrm{~d}$ & N.D. \\
Acacia milleriana & $118,09 \mathrm{c}$ & $85,46 \mathrm{~d}$ & $915,01 \mathrm{ab}$ & $427,18 \mathrm{c}$ & $285,10 \mathrm{c}$ & $35,03 \mathrm{a}$ & $73,72 \mathrm{a}$ \\
Acacia pennatula & $125,02 \mathrm{c}$ & $70,90 \mathrm{~d}$ & $929,05 \mathrm{a}$ & $590,81 \mathrm{a}$ & $358,05 \mathrm{a}$ & $28,72 \mathrm{~b}$ & $39,70 \mathrm{c}$ \\
Phitecollobium dulce & $196,13 \mathrm{~b}$ & $101,41 \mathrm{c}$ & $899,07 \mathrm{~b}$ & $452,99 \mathrm{~b}$ & $293,13 \mathrm{~b}$ & $6,00 \mathrm{c}$ & $0,00 \mathrm{e}$ \\
Genipa americana & $94,00 \mathrm{c}$ & $84,52 \mathrm{~d}$ & $915,10 \mathrm{ab}$ & $377,74 \mathrm{~d}$ & $309,52 \mathrm{~b}$ & $9,09 \mathrm{c}$ & $2,70 \mathrm{e}$ \\
Diphysa robinoides & $187,12 \mathrm{~b}$ & $117,71 \mathrm{~b}$ & $882,19 \mathrm{c}$ & $317,64 \mathrm{~d}$ & $232,44 \mathrm{bd}$ & $6,42 \mathrm{c}$ & $14,92 \mathrm{~d}$ \\
Leucaena collinsii & $201,47 \mathrm{ab}$ & $101,84 \mathrm{c}$ & $898,23 \mathrm{~b}$ & $275,00 \mathrm{e}$ & $191,01 \mathrm{c}$ & $3,01 \mathrm{~d}$ & $128,46 \mathrm{~d}$ \\
Erythrina goldmanii & $228,32 \mathrm{a}$ & $120,00 \mathrm{~b}$ & $880,43 \mathrm{c}$ & $431,50 \mathrm{bc}$ & $288,00 \mathrm{bc}$ & $6,82 \mathrm{c}$ & $1,54 \mathrm{e}$ \\
Promedio & 165,7 & 102,9 & 897,1 & 408,8 & 277,6 & 13,8 & 34,1 \\
Desviación estándar & $\pm 5,5$ & $\pm 2,0$ & $\pm 2,0$ & $\pm 8,9$ & $\pm 4,7$ & $\pm 1,2$ & $\pm 4,3$ \\
\hline
\end{tabular}

${ }^{1}$ PC: Proteína Cruda; Ce: Cenizas; MO: Materia Orgánica; FDN: Fibra Detergente Neutro; FDA: Fibra Detergente Ácida; FT: Fenoles Totales; TC: Taninos Condensados. Medias en la misma columna seguidas por diferentes letras son estadísticamente diferentes $(\mathrm{p}<0,05)$. ND: No Detectado. 
Cuadro 2. Composición química ( $\mathrm{g} / \mathrm{kg}$ de materia seca) de frutos de leñosas forrajeras del trópico seco del estado de Chiapas, México, 2004.

\begin{tabular}{llccclll}
\hline \multicolumn{1}{c}{ Especies } & \multicolumn{1}{c}{ PC } & Ce & MO & FDN & FDA & FT & TC \\
\hline Leucaena collinsii & $186,01 \mathrm{a}$ & $58,42 \mathrm{~b}$ & $942,09 \mathrm{a}$ & $519,10 \mathrm{~b}$ & $370,29 \mathrm{~b}$ & $13,12 \mathrm{~b}$ & $36,33 \mathrm{a}$ \\
Guazuma ulmifolia & $58,03 \mathrm{~b}$ & $53,55 \mathrm{~b}$ & $947,10 \mathrm{a}$ & $461,05 \mathrm{c}$ & $354,18 \mathrm{~b}$ & $6,04 \mathrm{c}$ & $2,80 \mathrm{c}$ \\
Acacia milleriana & $81,10 \mathrm{~b}$ & $50,63 \mathrm{~b}$ & $949,05 \mathrm{a}$ & $523,45 \mathrm{~b}$ & $372,30 \mathrm{~b}$ & $26,02 \mathrm{a}$ & $24,90 \mathrm{~b}$ \\
Enterolobium cyclocarpum & $164,32 \mathrm{a}$ & $43,10 \mathrm{~b}$ & $966,96 \mathrm{a}$ & $339,63 \mathrm{~d}$ & $221,55 \mathrm{c}$ & $1,41 \mathrm{c}$ & $3,40 \mathrm{c}$ \\
Ficus glabrata & $158,09 \mathrm{a}$ & $98,20 \mathrm{a}$ & $902,12 \mathrm{a}$ & $644,01 \mathrm{a}$ & $498,12 \mathrm{a}$ & $0,23 \mathrm{c}$ & $0,61 \mathrm{c}$ \\
Promedio & 129,45 & 60,73 & 941,41 & 497,22 & 363,11 & 9,34 & 13,52 \\
Desviación estándar & $\pm 5,65$ & $\pm 2,17$ & $\pm 2,48$ & $\pm 11,00$ & $\pm 9,87$ & $\pm 1,17$ & $\pm 1,61$ \\
\hline
\end{tabular}

PC: Proteína Cruda; Ce: Cenizas; MO: Materia Orgánica; FDN: Fibra Detergente Neutro; FDA: Fibra Detergente Ácida; FT: Fenoles Totales; TC: Taninos Condensados. Medias en la misma columna seguidas por diferentes literales son estadísticamente diferentes $(\mathrm{p}<0,05)$.

el caso de follajes (Cuadro 3) y de A. milleriana, L. collinsii y G. ulmifolia para el caso de frutos (Cuadro 4).

Al relacionar la composición química y la producción de gas sin PEG a 24 h se encontró que en el follaje la producción de gas se asoció con el contenido de FT y TC de las especies estudiadas $(-0,68, p<0,04$ y $-0,67$, $\mathrm{p}<0,03$, respectivamente), lo que indicaría la actividad antinutricional de estos compuestos. Se puede apreciar en los Cuadros 1 y 3 que las especies que incrementaron su producción de gas al utilizar PEG fueron aquellas cuyos contenidos de FT y TC eran altos. Los incrementos en la producción de gas fue resultado de la unión de estos compuestos antinutricionales al PEG inhibiéndose, con éste último, su actividad (Tolera et al. 1997). Para el caso de las especies que no presentaron incrementos en la producción de gas, podría explicarse por el hecho de que los efectos del PEG son específicos a los compuestos antinutricionales (i.e taninos) y que la ausencia de éstos o su nivel bajo presente provoca que el PEG no tenga ningún efecto sobre la fermentación y,

Cuadro 3. Producción de gas ( $\mathrm{ml} 500 \mathrm{mg} / \mathrm{MS})$ a $24 \mathrm{~h}$ de follaje de leñosas forrajeras del trópico seco del estado de Chiapas, México, 2004.

\begin{tabular}{lcccr}
\hline Especie & \multicolumn{2}{c}{ Vol. de gas a 24 h } & E.E.M.* & $\begin{array}{c}\text { Incremento } \\
(\%)\end{array}$ \\
\cline { 2 - 3 } & Sin PEG & Con PEG & & 37,20 \\
Guazuma ulmifolia & 51,77 a & $71,03 \mathrm{~b}$ & 2,8 & 2,84 \\
Gliricida sepium & $96,45 \mathrm{a}$ & $99,19 \mathrm{a}$ & 3,7 & 25,88 \\
Acacia milleriana & $55,56 \mathrm{a}$ & $69,94 \mathrm{~b}$ & 2,2 & 74,37 \\
Acacia pennatula & $39,41 \mathrm{a}$ & $68,72 \mathrm{~b}$ & 2,8 & 0,00 \\
Pithecellobium dulce & $73,30 \mathrm{a}$ & $72,82 \mathrm{a}$ & 2,0 & 4,64 \\
Genipa americana & 104,00 a & $108,83 \mathrm{a}$ & 2,6 & 7,30 \\
Diphysa robinoides & $99,22 \mathrm{a}$ & $106,47 \mathrm{a}$ & 3,2 & 33,04 \\
Leucaena collinsii & $53,63 \mathrm{a}$ & $71,35 \mathrm{~b}$ & 1,0 & 0,40 \\
Erythrina goldmanii & 89,00 a & $89,36 \mathrm{a}$ & 2,9 & \\
\hline \multicolumn{1}{c}{ Promedio } & 73,59 & 84,19 & & \\
\hline
\end{tabular}

*E.E.M.= Error estándar de las medias. Medias en la misma hilera seguidas por diferentes literales son estadísticamente diferentes $(\mathrm{p}<0,05)$. In cremento $(\%)=(($ volumen de gas con PEG $)-($ volumen de gas sin PEG $)) /$ (volumen de gas sin PEG)*100. 
Cuadro 4. Producción de gas (ml $\left.500 \mathrm{mg} \mathrm{MS}^{-1}\right)$ a $24 \mathrm{~h}$ de frutos de leñosas forrajeras del trópico seco del estado de Chiapas, México, 2004.

\begin{tabular}{|c|c|c|c|c|}
\hline \multirow[t]{2}{*}{ Especie } & \multicolumn{2}{|c|}{ Vol. de gas a $24 \mathrm{~h}$} & \multirow[t]{2}{*}{ EEM* } & \multirow{2}{*}{$\begin{array}{c}\text { Incre- } \\
\text { mento } \\
(\%)\end{array}$} \\
\hline & Sin PEG & Con PEG & & \\
\hline Leucaena collinsii & $79,81 \mathrm{a}$ & $85,86 \mathrm{~b}$ & 1,2 & 7,58 \\
\hline Guazuma ulmifolia & $102,02 \mathrm{a}$ & $110,99 \mathrm{~b}$ & 2,1 & 8,79 \\
\hline Acacia milleriana & $57,19 \mathrm{a}$ & $70,02 \mathrm{~b}$ & 2,0 & 22,43 \\
\hline $\begin{array}{l}\text { Enterolobium cyc- } \\
\text { locarpum }\end{array}$ & $129,83 \mathrm{a}$ & $138,24 \mathrm{a}$ & 4,3 & 6,47 \\
\hline Ficus glabrata & $26,35 \mathrm{a}$ & $26,15 \mathrm{a}$ & 1,7 & 0,00 \\
\hline Promedio & 79,04 & 86,25 & & \\
\hline
\end{tabular}

*EEM= Error estándar de las medias. Medias en la misma hilera seguidas por diferentes literales son estadísticamente diferentes $(\mathrm{p}<0,05)$. Incremento $(\%)=(($ volumen de gas con PEG $)-($ volumen de gas sin PEG)) / (volumen de gas sin PEG)*100

por tanto, en la producción de gas in vitro (Mlambo et al. 2002). Una ventaja de lo anterior es que las bajas o moderadas concentraciones de TC y/o FT en estas especies podrían incrementar la utilización de su proteína en rumiantes (Reed 1995), mientras que las especies con altas concentraciones son claramente detrimentales para los animales (Hervas et al. 2000).

De manera semejante a lo obtenido en este trabajo, se han reportado relaciones altas y negativas entre la producción de gas in vitro a $24 \mathrm{~h}$ y el contenido de polifenoles en especies arbóreas, sobre todo con los taninos condensados (Nsahlai et al. 1994, Tolera et al. 1997). En el análisis de regresión simple aplicado para describir la producción de gas a 24 h (sin PEG) en los follajes, únicamente los componentes fenólicos fueron considerados. La adición de otros componentes no mejoró la habilidad de predicción de la ecuación:

Gas producido a $24 \mathrm{~h}=97,5( \pm 7,9)-10,1( \pm 4,3)$ FT $-2,93( \pm 1,2)$ TC $(\mathrm{R}=71,8, \mathrm{P}<0,02)$

Para el caso de frutos, las relaciones fueron diferentes, ya que no se encontró correlación entre los componentes fenólicos (FT y TC) con la producción de gas in vitro a 24 h sin PEG. Sin embargo, ésta sí estuvo asociada a las fracciones de fibra (FDN= $-0,97$, $\mathrm{p}<0,006$ y $\mathrm{FDA}=-0,94, \mathrm{p}<0.01)$. Lo anterior explicaría el comportamiento del fruto $F$. glabrata, pues la producción de gas in vitro a 24 h fue la más baja de todos, sin embargo, la falta de respuesta al tratamiento con PEG (Cuadro 4) debido a sus valores bajos de TC y FT, conduce a señalar a sus fracciones de fibra como las responsables, las cuales fueron elevadas (Cuadro 2). Para el caso de frutos, la producción de gas a 24 h (sin PEG) se ajustó a la siguiente ecuación:

Gas producido a $24 \mathrm{~h}=253,0( \pm 25,2)-3,5$ $( \pm 0,49)$ FDN $\left(R^{2}=92,4, p<0,006\right)$

Gas producido a $24 \mathrm{~h}=218,0( \pm 30,0)-3,8$ $( \pm 0,8)$ FDA $\left(R^{2}=84,4, p<0,01\right)$

La falta de respuesta en los frutos podría deberse a que la respuesta biológica de los compuestos antinutricionales presentes en ellos dependen de su naturaleza y a sus variaciones entre las especies de plantas (Khazaal et al. 1994). Lo anterior enfatiza la importancia de determinar la estructura química, en adición a las cantidades de compuestos antinutricionales en las especies arbóreas, y ser usadas para predecir efectos biológicos de estos compuestos en la digestión ruminal (Ndlouv y Nherera 1997). Ésto indicaría también que es necesario identificar o trabajar con otros grupos de compuestos antinutricionales. Otra respuesta podría basarse en la tabla de composición química de los frutos bajo estudio (Cuadro 4), en dichos cuadros muestran especies con altas y muy bajas concentraciones de FT y TC, ésto podría dar como resultado que la relación entre la concentración de compuestos antinutricionales y la producción de gas no sea apropiada como ha sido reportada en otro trabajo (Khazaal et al. 1994), o bien al bajo número de frutos evaluados.

Las producciones de gas a $24 \mathrm{~h}$ obtenidas en este trabajo son mayores a las reportadas en arbóreas del Mediterráneo con o sin PVP (Polivinilpirrolidone, de acción similar al PEG) (Khazaal et al. 1994) Similar tendencia se observa en otro trabajo, al comparar estos datos con la producción de gas a $24 \mathrm{~h}$ con y sin PVP en arbóreas muestreadas durante dos estaciones del año (Hervas et al. 2000). Las diferencias probablemente se encuentren asociadas a las distintas familias evaluadas o a la cantidad de sustrato utilizada en cada uno de los trabajos. Comparando a las especies, se han reportado valores de producción de gas a $24 \mathrm{~h}$ en $G$. sepium de 31,8 ml (Apori et al. 1998), valor inferior al encontrado para esta especie en este trabajo $(96,4 \mathrm{ml})$. Por otro 
lado, los bajos valores informados y producidos a 24 $\mathrm{h}$ por diversas especies de Acacias reportados por Abdulzarak et al. (2000) concuerdan en este trabajo con los obtenidos para A. pennatula y A. milleriana.

Parámetros de degradación obtenidos por la producción de gas in vitro. El uso de PEG benefició $(\mathrm{p}<0,05)$ la producción potencial $(\mathrm{a}+\mathrm{b})$ de gas en $D$. robinoides, A. pennatula y A. milleriana, y solo la tasa los frutos de A. milleriana y G. ulmifolia, indicando una diferente actividad del PEG en el follaje y fruto arbóreo (Cuadro 5). Los valores encontrados en el presente artículo de parámetros de degradación en los follajes de $L$. collinsii y G. ulmifolia se encuentran dentro de los rangos reportados en otros trabajos (Menke y Steingass 1988).

La importancia que representa la producción de gas potencial $(\mathrm{a}+\mathrm{b})$ cuando los alimentos son incubados in vitro con líquido ruminal, está relacionada con la degradabilidad de éstos y, por tanto, con los valores de energía del alimento (Menke y Steingass 1988). Ante ello, el follaje de G. americana, G. sepium, D. robinoides y E. goldmanii y el fruto de E. cyclocarpum y G. ulmifolia, por sus valores de a+b (Cuadro 5) representan especies potenciales a ser estudiadas como fuentes importantes de energía. Por el contrario, las bajas producciones de gas liberado por los follajes de A. pennatula, A. milleriana, L. collinsii y G. ulmifolia y los frutos de $F$. glabrata y A. milleriana, confirman tener en consideración de que el valor energético del follaje arbóreo debe ser complementado con fuentes energéticas suficientes para hacer más eficiente la utilización de nutrientes, pues si bien, los árboles tienen efectos positivos sobre el aporte de $\mathrm{NH}_{3}$ al medio ambiente ruminal, en energía muchos de ellos resultan deficientes al igual que los pastos tropicales (D'Mello 1992). Las variaciones en la producción potencial de gas encontradas en las diferentes especies podrían resultar en consumos diferentes entre las especies cuando se ofrezcan como únicas en la dieta, debido a que esta producción está asociada con los niveles de FDN y TC en arbustos (Nsahlai et al. 1994).

Cuadro 5. Parámetros de fermentación obtenidos a través de la producción de gas in vitro $\left(\mathrm{ml} 500 \mathrm{mg} \mathrm{MS}^{-1}\right)$ con y sin -la utilización de PEG-4000 de leñosas forrajeras del trópico seco del estado de Chiapas, México, 2004.

\begin{tabular}{|c|c|c|c|c|c|c|c|c|c|c|}
\hline \multirow{2}{*}{$\begin{array}{l}\text { Especie } \\
\text { Follajes } \\
\end{array}$} & \multicolumn{5}{|c|}{ Sin PEG } & \multicolumn{5}{|c|}{ Con PEG } \\
\hline & $\mathrm{a}+\mathbf{b}, \mathrm{ml}$ & $\mathrm{a}, \mathrm{ml}$ & b, ml & c, $\mathbf{m l ~ h}^{-1}$ & rsd & $\mathrm{a}+\mathrm{b}, \mathrm{ml}$ & $\mathrm{a}, \mathrm{ml}$ & b, ml & $\mathrm{C}, \mathrm{ml} \mathrm{h}^{-1}$ & rsd \\
\hline L. collinsii & 137,25 & $-2,95$ & 140,20 & 0,021 & 2,8 & 139,63 & $-1,07$ & 140,70 & 0,028 & 3,8 \\
\hline D. robinoides & $171,63 \mathrm{a}$ & $-16,07$ & $187,73 \mathrm{a}$ & 0,037 & 5,1 & $179,06 \mathrm{~b}$ & $-17,94$ & $197,09 \mathrm{~b}$ & 0,039 & 8,9 \\
\hline G. sepium & 163,33 & $-11,57$ & 174,91 & 0,038 & 3,5 & 164,43 & $-9,57$ & 174,08 & 0,039 & 8,1 \\
\hline E. goldmanii & 158,27 & $-16,13$ & 174,45 & 0,037 & 4,4 & 159,22 & $-16,58$ & 175,84 & 0,037 & 6,5 \\
\hline G. americana & 188,33 & $-12,78$ & 201,16 & 0,035 & 5,6 & 191,26 & $-14,84$ & 206,12 & 0,037 & 4,6 \\
\hline P. dulce & 149,45 & $-3,15$ & 152,61 & 0,029 & 4,8 & 146,29 & $-4,21$ & 150,53 & 0,029 & 3,9 \\
\hline G. ulmifolia & 162,30 & $-6,20$ & 168,52 & 0,18 a & 3,8 & 156,76 & $-4,24$ & 161,05 & $0,026 \mathrm{~b}$ & 6,0 \\
\hline A. pennatula & $105,04 \mathrm{a}$ & $-2,36$ & $107,48 \mathrm{a}$ & $0,021 \mathrm{a}$ & 5,9 & $130,96 \mathrm{~b}$ & $-2,64$ & $133,66 \mathrm{~b}$ & $0,031 \mathrm{~b}$ & 4,4 \\
\hline A. milleriana & $130,74 \mathrm{a}$ & $-3,36$ & $134,18 \mathrm{a}$ & $0,024 \mathrm{a}$ & 3,5 & $143,29 \mathrm{~b}$ & $-7,01$ & $150,30 \mathrm{~b}$ & $0,029 \mathrm{~b}$ & 4,1 \\
\hline Promedio & 151,82 & 8,29 & 160,14 & 0,05 & & 153,98 & 8,68 & 165,49 & 0,03 & \\
\hline \multicolumn{11}{|l|}{ Frutos } \\
\hline A. milleriana & 120,98 & 1,88 & 119,15 & $0,026 \mathrm{a}$ & 4,4 & 122,64 & 0,24 & 122,42 & $0,034 \mathrm{~b}$ & 3,0 \\
\hline E. cyclocarpum & 193,88 & $-21,22$ & 215,11 & 0,049 & 6,9 & 196,36 & $-24,54$ & 220,94 & 0,053 & 8,8 \\
\hline L. collinsii & 164,35 & $-8,35$ & 172,72 & 0,028 & 3,8 & 168,34 & $-8,26$ & 176,63 & 0,030 & 3,5 \\
\hline G. ulmifolia & 165,25 & $-1,45$ & 166,73 & $0,040 \mathrm{a}$ & 6,1 & 152,18 & $-4,72$ & 156,93 & $0,055 \mathrm{~b}$ & 3,8 \\
\hline F. glabrata & 71,03 & $-1,43$ & 72,46 & 0,020 & 3,8 & 70,10 & $-2,68$ & 72,78 & 0,021 & 2,3 \\
\hline Promedio & 143,09 & 6,86 & 149,23 & 0,03 & & 141,92 & 8,08 & 149,94 & 0,03 & \\
\hline
\end{tabular}

Para cada parámetro de fermentación, medias en la misma hilera seguidas por diferentes literales son estadísticamente diferentes $(\mathrm{p}<0,05)$.

$\mathrm{a}+\mathrm{b}=$ volumen potencial de gas producido; $\mathrm{a}=$ fracción soluble; $\mathrm{b}=$ Volumen de gas producido con el tiempo; $\mathrm{c}=$ Velocidad de producción de gas. 


\section{CONCLUSIONES}

La producción de gas in vitro y la composición química de forrajes arbóreos del trópico seco del sur de México mostró un valor nutritivo potencial como alimento de rumiantes. Sin embargo, se detectó alta actividad de compuestos antinutricionales en L. collinsii, G. ulmifolia, A. pennatula y A. milleriana, resultando el PEG una herramienta útil como indicador del efecto de compuestos antinutricionales en estas especies.

\section{LITERATURA CITADA}

Abdulrazak, SA; Fujihara, T; Ondiek, JK; Orskov, ER. 2000. Nutritive evaluation of some Acacia tree leaves from Kenya. Animal Feed Science and Technology 85: 89-98.

Apori, SO; Castro, FB; Shand, WJ; Orskov, ER. 1998. Chemical composition, in sacco degradation and in vitro gas production of some Ghanaian browse plants. Animal Feed Science and Technology 76: 129-137.

Association of Official Agricultural Chemists. 1990. Official methods of analysis of the association of official agricultural chemists. 15 ed. Washington, DC USA. 390 p.

Devendra, C. 1990. The use of shrubs and tree fodder by ruminants. In: Devendra, C. ed. Shrubs and tree fodder for farm animals. Proceedings of workshop, 24-29 July 1989. Denpasar, Indonesia. International Development Research Centre. Ottawa, Canada. p. 214-245.

D'mello, JPF. 1992. Chemical constrains to the use of tropical legumes in animal nutrition. Animal Feed Science and Technology 38: 237-261.

Fick, KR; Mcdowell, LR; Miles, PH; Wilkinson, NS; Funk, JP; Conrad, JH; Valdivia, R. 1979. Métodos de análisis de minerales para tejidos de plantas y animales. Animal Science. Department. Universidad de Florida. USA. p. 80.

García, DE; Medina, GM. 2006. Composición química, metabolitos secundarios, valor nutritivo y aceptabilidad relativa de diez árboles forrajeros. Zootecnia Tropical. 24(3): 233-250
Getachew, G; Blummel, M; Makkar, HPS; Becker, K. 1998. In vitro gas measuring techniques for assessment of nutritional quality of feeds: a review. Animal Feed Science Technology 72: 261-281.

Hervas, L; Frutos, P; Ramos, G; Giraldez, FJ; Alvarez, DP; Mantecon, AR. 2000. Use of the gas production technique to estimate the nutritive value of condensed tannins-containing shrubs species from a mountain area in northern Spain. British Society of Animal Science (UK), Wageningen, University (NL) and IDTNO Animal Nutrition (NL). p. 60.

Khazaal, K; Boza, J; Orskov, ER. 1994. Assesment of phenolics related antinutritive effects in mediterranean browse: a comparation between the use of the in vitro gas production technique with or without insoluble polyvinylspolypyrrolidone or nylon bag. Animal Feed Science and Technology 49: 133-149.

Makkar, HPS; Singh, B. 1991. Distribution of condensed tannins (proanthocyanidins) in various fibre fractions in young and mature leaves of some oak species. Animal Feed Science and Technology 32: 253-260.

Makkar, HPS; Blummel, M; Becker, K. 1995. Formation of complexes between plyvinil pyrrolidones or polyethylene glycols and tannins and their implication in gas production and true digestibility in vitro techniques. British Journal of Nutrition 73: 897-913.

Makkar, HPS. 2003. Quantification of tannins in tree and shrubs foliage. A Laboratoy Manual. Klumer Academic Publisher. Netherlands. 102. p.

Menke, KH; Steingass, H. 1988. Estimation of the energetic feed value obtained from chemical analysis and in vitro gas production using rumen fluid. Animal Research and Development 28: 7-55.

Mlambo, V; Mould, FL; Mueller-harvey, I; Smith, T; Owen, E. 2002. In vitro fermentation characteristics of separated tree fruits components with added polyethylene glycol (PEG) as a diagnostic tool for effect of tannins. British Society of Animal Science (UK), Wageningen, University (NL) and IDTNO Animal Nutrition (NL). (en línea). Disponible en: http://www.bsas.org.uk/ downloads/annlproc/Pdf2002/132.pdf 
Mtengeti, EJ; Mhelela, A. 2006. Screening of potential indigenous browse species in semi-arid central Tanzânia. A case of Gairo division. Livestock Research for Rural Development (18) 8. (en línea). Disponible en: http:// www.cipav.org.co/lrrd/lrrd18/8/mten18108.htm

Mupangwa, JF; Ngongon, NT; Topps, JH; Ndlouv, P. 1997. Chemical composition and dry matter degradability profiles of forage legumes Cassia rotundifolia cv. Wynn, Lablab purpureus cv. Highworth and Macroptilium atropurpureum cv. Siritar at 8 weeks of growth (pre-anthesis). Animal Feed Science Technology 69: 167-178.

Ndlouv, LR; Nherera, FU. 1997. Chemical composition and relationships to in vitro gas production of Zimbabwe browsable indigenous tree species. Animal Feed Science and Technology 69:121-129.

Norton, BW. 1994. The nutritive value of tree legumes. In: Forage Tree Legumes in Tropical Agriculture. C. Gutteridge and H. Shelton. eds. CAB international, UK. p. 177-192.

Nsahlai, IV; Siaw, DE; Osuji, PO. 1994. The relationships between gas production and chemical composition of 23 browses of the genus Sesbania. J. of Sci. Food and Agric. 65: 13-20.

Pinto, RR; Ramírez, AL; Kú, VJC; Ortega, RL. 2002. Especies arbóreas y herbáceas forrajeras del sureste de México. Pastos y forrajes 25 (3): 171-180.

Pinto, RR. 2002. Árboles y arbustos con potencial forrajero del centro de Chiapas. Tesis Doctoral. Facultad de Medicina Veterinaria y Zootecnia. Universidad Autónoma de Chiapas 315 p.

Ramana, DBV; Sultan, S; Solanki, KR; Negi, AS. 2000 Nutritive evaluation of some nitrogen and non-nitrogen fixing multipurpose tree species. Animal Feed Science and Tecnology 88 (1-2): 103-111.
Reed, JD. 1995. Nutritional toxicology of tannins and related polyphenols in forage legumes. J. Anim. Sci. 73:15161528.

Salem, ZM; Salem, MZM; El-Adawy, MM; Robinson, PH. 2006 Nutritive evaluations of some browse tree foliage during the dry season: secondary compounds, feed intake and in vivo digestibility in sheep and goats. Animal Feed Science and technology 127(3-4): 251-267.

SAS (Statistical Analysis System). 1999. User's guide: Statistics [CD-ROM Computer file]. North Carolina, USA. Version 8. SAS Inst. Inc., Cary, NC.

Sosa, EE; Pérez, RD; Ortega, RL; Zapata, BG. 2004. Evaluación del potencial forrajero de árboles y arbustos tropicales para la alimentación de ovinos. Técnica Pecuaria en México 42(2): 129-144.

Steel, RG; Torrie, JH. 1988 Bioestadística. Principios y Procedimientos. 2 ed, McGraw Hill. México DF. 371 p.

Tedonkeng, EP; Boukila, B; Fonteh, FA; Tendonkeng, F; Kana, JR; Nanda, NS. 2007. Nutritive value of some grasses and leguminous tree leaves of the Central region of Africa. Animal Feed Science and Tecnology 135 (3-4): 273-282.

Theodorou, MK; Williams, BA; Dhanoa, MS; Mcallan, AB; France J. 1994. A simple gas production method using a pressure transducer to determine the fermentation kinetics of ruminant feeds. Animal Feed Science and Technology 48: 185-197.

Tolera, A; Khazaal, K; Orskov, ER. 1997. Nutritive evaluation of some browse species. Animal Feed Science Technology 67: 181-195.

Van Soest, PJ; Robertson, JB. 1991. Analysis of forages and fibrous foods. Cornell University. E.U. Laboratory manual for Animal Science. No 613. p. 165. 
